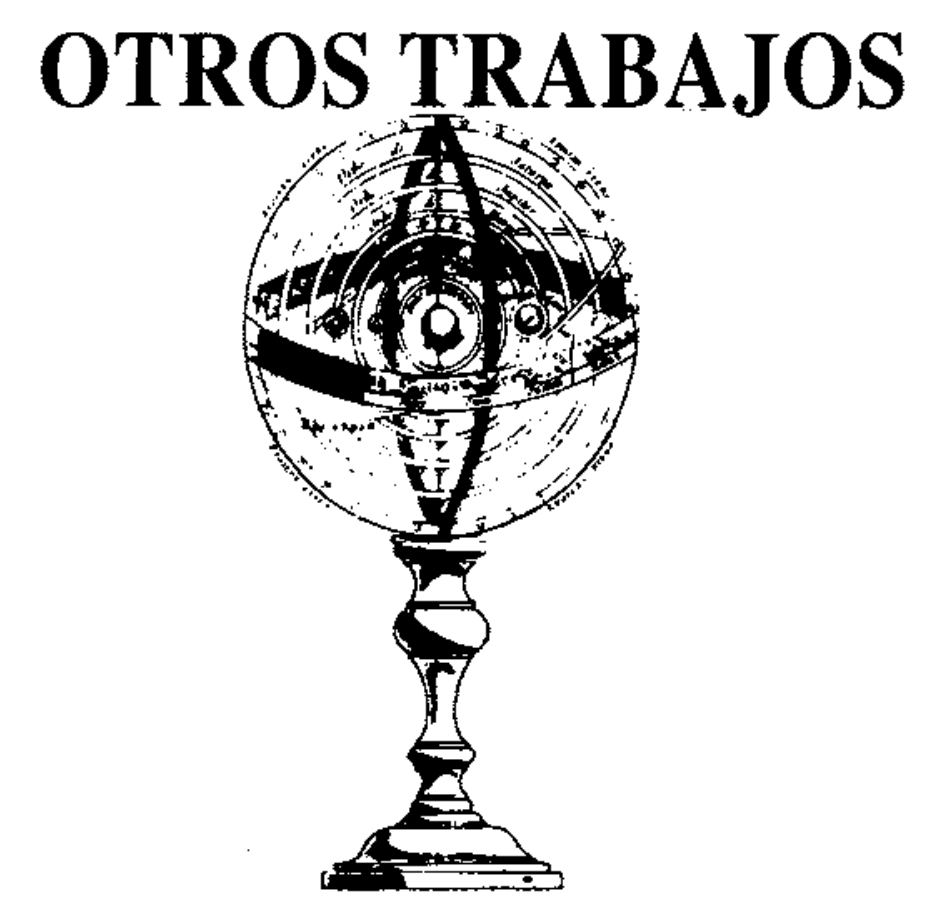

\title{
PROBLEMAS DE TERMINOLOGÍA EN ESTUDIOS REALIZADOS ACERCA DE «LO QUE EL ALUMNO SABE» SOBRE CIENCIAS
}

JIMÉNEZ GÓMEZ, E., ${ }^{1}$ SOLANO MARTÍNEZ, I. ${ }^{1}$ y MARÍN MARTÍNEZ, N. ${ }^{2}$

${ }^{1}$ Departamento de Didácticas de las Ciencias Experimentales. Universidad de Murcia.

${ }^{2}$ Departamento de Didácticas de las Ciencias Experimentales. Universidad de Almería.

\section{SUMMARY}

In this paper we have carried out a bibliographic review of the terms currertly used by science education researchers to describe «pupils' knowledge» of Science. Further, we have analised their meanings from the perspective of the methodological framework used and have offered our own proposal, as a result of that review, so as to reach a consensus on both the terminology and the possible range of meanings to be used. It must be pointed out that we have excluded those studies which discuss «pupils' knowledge» from a purely psychological perspective because of the enormous amount of bibliography on this topic.

\section{INTRODUCCIÓN}

Un intento de acomodar los procesos de enseñanza a las peculiaridades cognoscitivas del alumno pasa por conocer,

por parte del investigador, «lo que el alumno sabe» sobre los contenidos de ciencias a enseñar. Dicha acomoda- 
ción es todavía un problema pendiente de resolver; sin cmbargo su estudio ha permitido:

1. Mejorar la comunicación entre los profesores y alumnos durante el desarrollo del acto didáctico (Driver 1989).

2. Realizar nuevos discños de enseñanza (Osborne y Freyberg 1985, Posner et al. 1982, Nussbaum y Novick 1981 , entre otros).

3. Ayudar a conocer mejor los procesos de aprendizaje.

4. Crear una nueva perspectiva sobre el aprendizaje (Driver y Oidhan 1985, Glaser y Bassok 1989, entre otros).

5. Ayudar al profesor a interpretar los sucesos que tienen lugar en el aula y especialmente en la toma de decisiones.

El papel que juega "lo que el alumno sabe» sobre un determinađo contenido en el diseño del proceso de enscñanza ha llevado a gran númcro de investigadores a realizar rrabajos sobre su identificación y descripción, fundamentación epistemológica, naturaleza, metodología de la investigación, etc. (Carmichael y otros 1990). Sin embargo, a pesar de la proliferación de trabajos aparecidos en los últimos años acerca de este tema, y desde la publicación de Gilbert y Watts (1983), poco se ha avanzado en clarificar su epistemologia y estatus ontogenético (Abimbola 1988, Gunstone 1989, entre otros\}, ni tan siquiera en unificar los términos y sus significados a la hora de referirnos a ello.

Con el fin de comprender la razón de la diversidad de términos y significados empleados, junto con el propósito inicial de realizar una propuesta đe unificación que podría consensuarse si se estima conveniente, para aplicar los mismos significantes a la hora de referirnos a «lo que el alumno sabe», se ha procedido a realizar una revisión bibliográfica de los términos utilizados por los distintos investigadores y educadores en ciencias, analizando el significado que le dan a éstos a partir del marco metodológico utilizado.

La extensa bibliografía existente sobre las explicaciones de los alumno en el dominio de las ciencias experimentales (química, física, biología y geología), sugiere establecer algunos criterios:

a) Se han seleccionado aquellos autores que han sido más referenciados, tanto en revistas de lengua española como anglosajona.

b) Para cada uno de los términos se ha seguido tuna búsqueda bibliográfica con la intención de encontrar aquellos trabajos donde el autor trata el significado de un determinado término de forma explícita y precisa.

c) Razories de extensión justifican dejar de lado los trabajos que tratan las explicaciones de los alumnos desde un marco estrictamente psicologico que podría ser objeto de un próximo trabajo.
Con la revisión bibliográfica se pretende analizar las razones que han llevado a los distintos autores a utilizar diferentes términos y significados para referirse a alo que el alumno saben. Finalmente, en base a las convergencias, simititudes y regularidades encontradas se realiza una propuesta.

\section{ASPECTOS METODOLÓGICOS Y TEÓ- RICOS UTILIZADOS EN LOS ESTUDIOS SOBRE «LO QUE EL ALUMNO SABE»}

Cuatro han sido los aspectos de interés sobre los que se ha centrado el análisis bibliográfico realizado, que se encuentran resumidos y ordenados en columnas para cada autor o grupo de autores en el anexo de este trabajo. Éstos han sido:

a) Terminología utilizada por los diferentes autores, los cuales se han ordenado de forma cronológica.

b) Significados asignados a cada uno de los términos por los distintos autores.

c) Contexto experimental que han utilizado los autores en sus investigaciones. Esto se ha utilizado principalmente para comprender la razón por la que unos autores utilizan un término o han propuesto otro nuevo.

d) Forma de categorizar las explicaciones de Ios alumnos, lo que ha permitido comprender la intencionalidad del trabajo realizado y, por tanto, mejorar la comprensión del análisis de los resultađos y sus implicáciones a la hora de establecer significados a los términos propuestos.

Del análisis de las dos últimas columnas del anexo se infieren una serie de regularidades, entre las que destacan las siguientes:

1. En la mayoría de los trabajos se realizan entrevistas personales (10 de los 18 referenciados en el anexo), donde se plantean al sujeto una scrie de situaciones o succsos en ciencias.

2. De las respuestas de los alumnos se identifican elementos comunes, se categorizan y, en la mayoría de los casos (14 de 18 ), se jerarquizan dichas categorías.

3. La jerarquización se realiza en función del acercamiento o separación que el investigador o educador piensa que existe entre las respuestas de los alumnos y la dada por la comunidad científica.

4. Son escasos los trabajos realizados sobre la evolución ontogenética de la fenomenología tratada. Publicaciones como la de Albert (1978) son una excepción.

5. Los artículos revisados hacen hincapié en el plano observable (formado por los sucesos o fenómenos físicos presentados, las preguntas realizadas por cl educador y las respuestas del sujeto), olvidándose del no observable (constituido por los elcmentos cognoscitivos que utiliza el sujeto y los mecanismos de interrelación entre ellos). Utilizan una metodología inductivista don- 
de el investigador posee como único esquema de búsqueda los que se derivan de la ciencia o de la historia de la ciencia (Marín y Jiménez Gómez 1992a).

6. De los trabajos referenciados ninguno interpreta las explicaciones de los alumnos, sólo se identifican y describen regularidades. Ello puede ser consecuencia del contex to teórico que utilizan los diferentes autores a la hora de analizar las respuestas de los alumnos, lo cual lleva a evaluar el conocimiento del alumno por las discrepancias o similitudes que presenta con el conocimiento académico referido al contenido objeto de la investigación.

De los trabajos revisados, destaca el hecho de que, siendo las respuestas de los alumnos fruto de su estructura cognoscitiva, pocas son las incursiones en el plano de la psicología como ocurre con los trabajos de Hibbard y Novack (1975) que utilizan la psicología de Ausubel; Nussbaum y Sharoni-Dagan (1983) la de Piaget, pero en este último caso es más una referencia superficial que una aplicación rigurosa de este marco teórico.

De todo lo anterior se infiere que Ios investigadores analizan las respuestas de los alumnos desde la propia ciencia. Si se tiene en cuenta que éstas provienen de la estructura cognoscitiva, es decir, del plano no observable, es necesario realizar estudios que integren ambos planos y que permitan su interpretación (Marín y Jiménez Gómez 1992a).

\section{TÉRMINOS Y SIGNIFICADOS UTILI- ZADOS EN LOS ESTUDIOS DE «LO QUE EL ALUMNO SABE»}

Los términos utilizados por los investigadores surgen de la propia metodología đe investigación. Así, se puede comprobar como Nussbaum y Novack (1976) necesitan un término, que denominan noción, para referirse al constructo conceptual realizado por el investigador inferido de las respuestas de los alumnos. Brumby (1979) encuentra que en las respuestas de los alumnos existen problemas de comprensión, por lo que se refiere a ellas con el nombre de misundertandings (fallos de comprensión). Otros, como Erickson (1979), encuentran ideas comunes en las respuestas de los alumnos, por lo que las denomina ideas compuestas. De forma análoga van apareciendo los 16 términos que han sido analizados, junto a sus significados, y que se exponen en el anexo.

Los significados, que son utilizados como atributos asociados a las explicaciones de los alumnos, se caracterizan porque:

a) Tienen carácter personal y están referidos a un tópico en concreto (Erickson 1979).

b) Evolucionan a través de la interacción con nuevos conocimientos (Hibbard y Novack 1975).

c) Son diferentes a los que asume la comunidad científica e inhiben el aprendizaje (Nussbaum y Sharoni-
Dagan 1983, Brumby 1979, Caramazza et al. 1981, Meheut et al. 1985, Viennot 1979).

d) Presentan r zgularidades (son repetibles y generalizables) para un nismo sujeto. Así, el entrevistado realiza el mismo tipo kle explicación a preguntas sobre contenidos científicos en diferentes contextos (Deadman y Kelly 1978, Caramazza et al. 1981, Viennot 1979, Meheut et al. 1985 ).

e) Suelen vari ar desde meras respuestas de percepción a modelos más elaborados, aunque suelen ser distorsionados con respecto ;. Ios que poseen los científicos (Novick y Nussbaum 1978).

f) Suelen ser comunes a los diferentes sujetos entrevistados (Erickson 1979, Viennot 1979).

g) Ofrecen un perfil conceptual sobre una determinada fenomenología (Erickson 1980).

h) Responden a modelos subjetivos, son intuitivos y erróneos (Car.łmazza et al. 1981, Viennot 1979).

i) Son consecuencia de la creatividad e imaginación, experiencia clirecta e interacción social del sujeto (Driver 1981).

La alta varialsilidad de significados asociados a los términos empieados por los diferentes autores se debe, en parte, al gran número de factores que intervienen en las explicaciones de los alumnos. Entre otros destacan:

a) En el plano observable: la dependencia del contexto, el tipo de prę̧unta realizada, número de variables que intervienen en la situación f́́sica presentada, etc.

b) En el plano no observable: los elementos de la estructura cognoscitiva y las interrelaciones entre dichos elementos, intereses, motivaciones de los alumnos, etc.

Con el fin de identificar regularidades, se van a separar los términos cue hacen referencia al plano observable del no observable. Este trabajo se refiere solo a los primeros, por allo se han excluido del análisis posterior los términos estructura cognitiva de Hibbard y Novack (1975), creencias ingenuas de Caramazza y otros (1981) y concepto de Albert (1978) (ver anexo), cuyo estudio entra dentro dŁl contexto de las teorías psicológicas, que serán objeto de un nuevo trabajo.

El resto de térninos hace referencia a las explicaciones de los alumno: o a interpretaciones que de éstas hace el investigador. Del anexo se inffiere que:

1. Si el análisis se realiza desde un plano más concreto, es decir, desde: la propia explicación, se observa que:

a) Las explicaciones verbales o icónicas no tienen ningún tipo de caracterización, es decir, son meras respuestas del sujeto que en la mayoría de los casos vienen influencias por los aspectos figurativos de la tarea (Novick y Nussbaum 1978, Deadman y Kelly 1978). 
b) Las explicaciones cumplen determinadas características, es decir:

- Presentan cierto grado de estabilidad (Meheut et al. 1985, Viennot 1979).

- Son respuestas elaboradas (Deadman y Kelly 1978).

- Presentan regularidades a pesar de modificar el contexto (Deadman y Kelly 1978).

- Son repetibles y generalizables a diferentes situaciones físicas (Caramazza et al. 1981).

Los autores para referirse a este tipo de explicaciones de los alumnos utilizan términos tales como: esquemas explicativos (Meheut et al. 1985) y razonamiento espontáneo (Viennot 1979), si bien este último término se define en función del primero (ver anexo).

2. Si el investigador las interpreta, éste genera un modelo sobre los conocimientos que posee el alumno sobre una determinada fenomenología. Los autores para referirse al perfil de conocimientos del sujeto introducen términos en función de que:

a) Se realice un análisis desde las discrepancias o similitudes entre el contenido del alumno y el académico. Desde esta perspectiva se han utilizado términos tales como: conceptos erróneos (Brumby 1979, Caramazza et al. 1981), ideas falsas (Nussbaum y Sharony-Dagan 1983, Meheut et al. 1985) y preconceptos (Carrascosa y Gil 1985).

b) Exista una mala comprensión del contenido académico, aquí aparece el término de fallos de comprensión (Brumby 1979).

c) Se comparen unas explicaciones o razonamientos de unos sujetos con otros. Aquí, los autores proponen los términos: ideas compuestas (Erickson 1979), razonamientos espontáneos (Viennot 1979) y creencias de los alumnos (Erickson 1980).

d) Consideren que son formas de ver el mundo que nos rodea diferentes a como lo hace la comunidad cientiffica. En tal caso, aparecen los términos: esquemas alternativos (Driver 1981), creencias de los alumnos (Erickson 1980), nociones (Nussbaum y Novak 1976, Nussbaum. 1979, Mali et al. 1979, Nussbaum y Sharony-Dagan 1983), concepciones (Deadman y Kelly 1978, Novick y Nussbaum 1978), ideas de los niños (Erickson 1979) y preconcepciones (Novick y Nussbaum 1981). Algunos autores, como García Hourcade y Rodríguez de Ávila (1985) diferencian entre preconcepciones y esquemas alternativos. Así, para ellos las opiniones o los enunciados observacionales son analizados por el investigador buscando las preconcepciones. De ellas, se obtiene un cuerpo teórico elemental que pueđe explicitarse y ser asumido de mancra consciente por los alumnos, lo que constituye un esquema alternativo, obtenieñdose sólo en fenomenologías concretas, como por ejemplo el calor y la mecánica, pero no en todo el contenido que se imparte en el proceso educativo (García Hourcade y Rodríguez de Ávila 1988).
Las diferencias que se detectan entre todos los términos provienen fundamentalmente del contenido de enseñanza objeto de la investigación y del marco teórico que cada investigador utiliza a la hora de llevar a cabo sus investigaciones (Abimbola 1988, Gilbert y Watts 1983, entre otros).

De lo anterior se infieren dos ideas que merecen ser resaltadas:

1. Si se hace referencia, en primer lugar, al tipo de explicación en las que subyace el mismo contenido físico, se encuentra que pueden ser, en una primera aproximación, de dos formas diferentes:

a) Repetibles y generalizables a pesar de cambiar el contexto, el tipo de pregunta, Ios aspectos figurativos de Ia tarea u otras variables; son respuestas elaboradas y tienen cierto grado de estabilidad.

b) Variables y dependientes del contexto, de los aspectos figurativos; en general son respuestas poco elaboradas, por Io que carecen đel carácter repetible y generalizable que caracteriza a las explicaciones del apartado anterior.

2. Las explicaciones son utilizadas por el investigador para construir un modelo acerca de los conocimientos que tienen los alumnos sobre una determinada fenomenología.

A los puntos anteriores se pueden añadir como características fundamentales de las explicaciones de los alumnos, además de las reseñadas anteriormente, las de ser diferentes a las aceptadas por Ia comunidad cientiffica y ser comunes a varios sujetos.

Por último, se quiere indicar que no se ha hecho un análisis exhaustivo de todos términos que los autores han utilizado. Asf́, Glad (Millar 1989) ha llegado a contabilizar 21. No obstante, se piensa que la revisión es suficientemente amplia para cubrir los objetivos del presente trabajo.

\section{DELIMITACIÓN DEL TÉRMINO CON- CEPCIÓN}

Las explicaciones de los alumnos, como se ha dicho anteriormente, pueden ser de dos tipos:

\section{Tipo 1}

Caracterizadas por tener el carácter de:

a) Repetición, es decir que la explicación no varía a pesar de modificar la situación física o el contexto en el que se presente, pero en las que subyace análogo contenido.

b) Generalizacion, si se amplia el campo de aplicación a nuevas situaciones.

c) Diferenciación, si el alumno discrimina o reconoce los contenidos en las diferentes situaciones físicas presentadas. 
Las caracterizaciones anteriores en las respuestas o explicaciones de los alumnos implican cierto grado de estabilidad (Meheut et al. 1985, Viennot 1979), son respuestas elaboradas (Deadman y Kelly 1978), presentan regularidades a pesar de modificar el contexto (Deadman y Kelly 1978) y son repetibles y generalizables en diferentes situaciones físicas (Caramazza et al. 1981).

En este caso, los términos utilizados por investigadores para referirse a este tipo de explicaciones según la revisión bibliográfica realizada son (ver anexo): esquema explicativo, término propuesto por Meheut y otros (1985) y el de razonamiento espontáneo, propuesto por Viennot (1979), si bien éste último se define en función del primero. De aquí, que se proponga el término esquemas explicativos como el más adecuado de los referenciados en el anexo y con el significado señalado anteriormente.

A partir de los esquemas explicativos, el investigador o educador estabiece el perfil conceptual y procedimental sobre una determinada fenomenología. En este caso se está haciendo una interpretación que va a depender del contexto teórico que utilice el investigador, de los objetivos del trabajo y de su propia estructura cognoscitiva (Gunstone 1989). Esto podría ser una de las causas que explican la diversidad de términos que aparecen cuando el educador o investigador se refiere a «lo que el alumno sabe».

Quizás más que consensuar el significante, habría que buscar convergencias atendiendo al significado. Así, se deberian establecer las bases de interpretación de las explicaciones de los alumnos por parte del investigador. Por ejemplo, algunos investigadores están interesados en el perfíl conceptual y se olvidan de aquellos aspectos relacionados con los procedimientos. De igual forma, a veces, se interpretan las explicaciones en términos de sus atributos negativos, por ejemplo cuando se dice que el $80 \%$ de los alumnos no tiene el concepto de energía. Otras veces sólo se tiene en cuenta el contenido del alumno que está relacionado con el contenido académico $y$, sin embargo, no se tienen en cuenta los esquemas asimiladores de dicho contenido; de aquí, que la interpretación que estâ haciendo el investigador sobre lo que sabe el alumno sobre ciencias es parcial y restrictiva.

Si los educadores e investigadores se ponen de acuerdo en el significado de la interpretación de las explicaciones de los alumnos, sería más fácil llegar a un consenso sobre el término a utilizar para designar el perfil conceptual y procedimental de aquéllos. En este sentido, varias han sido las propuestas que se exponen en el anexo, entre las que destacan los términos de noción, concepción, puntos de vista, esquemas alternativos y conceptos erróneos.

Entrar en las raíces de los términos anteriores y sus significados podría constituir un nuevo trabajo. Mientras esto se realiza, nosotros venimos razonando de la siguiente manera:

A partir de los esquemas explicativos, el investigador o educador puede obtener la mayor parte de la información significativa de los alumnos. Entendiendo por información significativa todo aquello que es relevante y ayuda a realizar provesos de enseñanza adecuados a las características psicológicas de los alumnos. Está constituido por:

a) El conocimiento conceptual que, a su vez, podría ser de dos tipos:

- relativo al contenido académico que se quiere enseñar;

- no relacionado con el contenido académico que se quiere enseñar y que es exclusivo de la ontogénesis del sujeto (Marín y Jiménez Gómez 1992b).

b) El nivel cognoscitivo, determinado por la capacidad operatoria de.. sujeto.

c) Las reglas dizasimilación de los esquemas cognoscitivos.

Para referirnos a la interpretación que el investigador 0 educador realiza para referirse a los conocimientos conceptuales que tiene el alumno sobre una determinada fenomenología, deducido de las reacciones del sujeto a situaciones fí jicas presentadas y que tienen relación con el contenido a enseñar, solemos utilizar el término concepción

\section{Tipo 2}

Son explicaciones que no cumplen aiguno de los requisitos del tipo 1 (repetición, generalización y diferenciación) y son, por tant ${ }^{\prime}$, dependientes del contexto, de los aspectos figurativos, de las variables que intervienen en el suceso, etc.; :se podrían distinguir, a su vez, dos tipos:

a) Las que of recen algún tipo de información significativa del alumno.

b) Las que scin meras repuestas de compromiso, que el sujeto realiza porque el educador así se lo exige o porque la exigencia de la tarea no está acomodada a la capacidad de respuesta del alumno, siendo este tipo de respuesta la que menos interés tiene a la hora de obtener informacion significativa del alumno.

Por último cabe indicar que en el plano no observable es donde se encijentra el origen de las explicaciones y, por tanto, de las concepciones. Es necesario aplicar un marco teórico y una metodología muy diferente de la que aparece en los trabajos referenciados en el anexo para poder relacionar los diferentes tipos de explicaciones con los elementos que integran la estructura cognoscitiva (esquemati de acción, representaciones, operaciones mentales, etc.). De aquí, que sea necesario, por tanto, realizar hipotesis sobre los elementos cognoscitivos y las interrelactones que puedan darse entre ellos, para poder comprinder las diferentes respuestas de los alumnos ante diferentes problemas planteados. A partir de aquí, habría que delimitar aquella información significativa que permita acomodar los procesos de enseñanza a las características psicologicas del sujeto y, en definitiva, a optimizar el aprendizaje.

Desde el punto de vista metodológico se requiere, entre otras cosas: c) elegir un contenido que tenga significado 
para los alumnos; $b$ ) determinar los factores que afectan a las situaciones o fenómenos físicos que se eligen para presentarlo a los alumnos, así como estudiar el carácter de sus explicaciones y, a partir de aquí, proceder a la identificación, descripción e interpretación de las concepciones (Marín y Jiménez Gómez 1992a).

Actualmente venimos trabajando tanto en el marco teórico como en el metodológico y se espera ofrecer, en un futuro no muy lejano, nuevos resultados.

\section{REFERENCIAS BIBLIOGRÁFICAS}

ABIMBOLA, I.O., 1988. The problem of terminology in the study of student conceptions in Science, Science Education, Vol. 72(2), pp. 175-184.

ALBERT, E., 1978. Development of the concept of heat in children, Science Education, Vol. 62(3), pp. 389-399.

BRUMBY, M., 1979. Problems in learning the concept of natural selection, Journal of Biological Education, Vol. 13(2), pp. $119-122$

CARAMAZZA, A., MCCLOSKEY, M. y GREEN, B., 1981. Naive beliefs in "sophisticated» subjects: misconceptions about trajectories of objects, Cognition, Vol. 9, pp. 117-123.

CARMICHAEL, P., DRIVER, R., HOLDING, B., PHILLIPS, I., TWIGGER, D. y WATTS, M., 1990. Research on Students' Conceptions in Science: a Bibliography, CSSME, University of Leeds (Leeds).

CARRASCOSA ALIS, J. y GIL PÉREZ, D., 1985. «La metodologia de la superficialidads y el aprendizaje de las ciencias, Enseñanza de las Ciencias, Vol. 3(2), pp. 113-120.

DEADMAN, J. y KELLY, P.J., 1978. What do secondary school joys understand about evolution an heredity before they art: taught the topics, Journal of Biological Education, Vol. 12(1), pp. 7-15.

DRIVER, R., 1981. Pupils' alternative frameworks in science, European Journal Science Education, Vol. 3(1), pp. 93-101.

DRIVER, R., 1989. Students' conceptions and the learning of science, International Journal Science Education, Vol. 11 , Special Issue, pp. $481-490$.

DRIVER, R. y OLDHAM, V., 1985. A Constructivist Approach to Curriculum Development in Science. Paper prepared for the Symposium Personal Construction of Meaning Educationa Secings, BERA, Shefield

ERICKSON, G.L., 1979. Children's conceptions of heat and temperature, Science Education, Vol. 63(2), pp. 221-230.

\section{NOTA}

Esperamos con este trabajo haber delimitado mejor algunos de los términos utilizados en la bibliografía actual para referirse a «lo que el alumno sabe». Por otro lado, serfa aconsejable que los autores hagan explícito en sus trabajos el significado de los mismos, en aras de una mejor comunicación entre educadores e investigadores en didáctica de las ciencias.
ERICKSON, G.L., 1980. Children's viewpoints of heat: a second look, Science Education, Vol. 64(3), pp. 323-336.

GARCÍA HOURCADE, J.L. y RODRIGUEZ DE ÁVILA, C., 1985. Preconcepciones del calor en $2^{\circ}$ de BUP, Enseñanza de las Ciencias, 3(3), pp. 188-193.

GARCÍA HOURCADE, I.L. y RODRÍGUEZ DE ÁVILA, C., 1988. Ideas previas, esquemas alternativos, cambio conceptual y trabajo en el aula, Enseñanza de las Ciencias, Vol. 6(2), pp. 161-166.

GILBERT, J.K. y WATTS, D.M., 1983. Concepts, misconceptions and alternative conceptions: changing perspectives in Science Education, Studies in Science Education, Vol. 10, pp. 61-98.

GLASER, R. y BASSOK, M., 1989. Learning Theory and the Study of Instruction, Ann. Rev. Psychol, 40, pp. 631-666.

GUNSTONE, R.F. y WHITE, R.T., 1981. Understanding of gravity, Science Education, Vol. 65(3), pp. 291-299.

GUNSTONE, R., 1989. A comment on the problem of terminology in the Study of Student Conceptions in Science, Science Education, Vol. 73(6), pp. 643-647.

HIBBARD, K.M. y NOVAK, I.D., 1975. Audio-Tutorial elementary school science instruction as a method for study of children's concept learning: particulate nature of matter, Science Education, Vol. 59(4), pp. 559-570.

KLEIN, C.A., 1982. Children's concepts of the Earth and the Sun: a cross cultural study, Science Education, Vol. 65 (1), pp. 95-107.

MALI, G.B. y HOWE, A., 1979. Developement of Earth and gravity concepts among Nepali children, Science Education, vol. 63(5), pp. 685-691.

MARÍN, N, y JIMÉNEZ GÓMEZ, E., 1992a. Problemas metodologicos en el tratamiento de tas concepciones de los alumnos en el contexto de la filosofía e historia de la ciencia, Enseñanza de las Ciencias, Vol. 10(3), pp. 335-339. 
MARÍN, N. y JIMÉNEZ GÓMEZ, E., 1992b. Contenido académico y contenido del alumno. Comunicación presentada en XIII Encuentros de Didáctica de las Ciencias Experimentales. Guadalajara.

MEHEUT, M., SALTIEL, E. y TIBERGHIEN, A., 1985. Pupils' (11-12 year olds) conceptions of combustion, European Journal Science Education, Vol.7(1), pp. 83-93.

MILLAR, R., 1989. Constructive Criticisms, International Joumal Science Education, Vol. 11, Special Issue, pp. 587-596.

NOVICK, S. y NUSSBAUM, J., 1978. Junior High School pupils' understanding of the particulate nature of matter: an interview study, Science Education, Vol. 3, pp. 273-281.

NOVICK, S. y NUSSBAUM, I, 1981. Pupils' understanding of the particulate nature of matter: a cross-age study, Science Education, Vol. 65(2), pp. 187-196

NUSSBAUM, J., 1979. Children's conceptions of de Earth as a cosmic body: a cross age study, Science Education, Vol. 63(1), pp. 83-93.
NUSSBAUM, J. y NOVAK, I.D., 1976. An assessment of children's ccncepts of the Earth utilizing structured interviews, Science Education, Vol. 60(4), pp. 535-550.

NUSSBAUM, J. y NOVICK, S., 1981. Brainstorming in the classroom ti invent a model: a case study, School Science Review, 221, p. 62.

NUSSBAUM, I. y SHARONI-DAGAN, N., I983, Changes in second grade childrens' preconceptions about the Earth as a cosmic body resulting from a short series of audio-tutorial lessons, Sci ence Education, Vol. 67(1), pp. 099-114.

OSBORNE, R. y FREYBERG, P., 1985. Learning in Science, "The implicution of childrens' science». (Heineman: Londres).

POSNER et al., 1982. Accommodation of a Scientific Conception: Toward a Theory of Conceptual Change, Science Education, Vol. 66, pp. 211-227.

VIENNOT, L., 1979. Spontaneous reasoning in elementary dynamics, European Journal Science Education, Vol. 1(2), pp. 205-221. 


\begin{tabular}{|c|c|c|c|c|}
\hline AUTORES & SIGNIFICANTE & SIGNIFICADO & CONTEXTO EXPERIMENTAL & CATEGORIZACIÓN \\
\hline $\begin{array}{l}\text { Hibbard, K M. y } \\
\text { Novak, J.D. } \\
1975 .\end{array}$ & $\begin{array}{l}\text { Estructura cognitiva } \\
\text { en una cierta área de } \\
\text { conocimiento } \\
\text { (cognitive estructure). }\end{array}$ & $\begin{array}{l}\text { Lo que subyace en las respurestas de } \\
\text { los individuos y que hace que éstos } \\
\text { respondan de igual forma ante } \\
\text { tareas que, siendo diferentes, lievan } \\
\text { implicito el mismo contenido } \\
\text { acadénico. Evoluciona a través de } \\
\text { la interacción de nuevos } \\
\text { conocimientos. }\end{array}$ & $\begin{array}{l}\text { Muestra: Jer grado de escuelas } \\
\text { elementales ( } 84 \text { con instrucción } \\
\text { audiotutorial y } 38 \text { no instruidos). } \\
\text { Contenido: Naturaleza particulada } \\
\text { de la materia. Preguntas abiertas } \\
\text { (produccion), de reconocimiento y } \\
\text { entrevistas. }\end{array}$ & $\begin{array}{l}\text { Obtienen respuestas que categorizan } \\
\text { de acuerdo con ia distancia o similitud } \\
\text { con el contenido académico. Utiliza la } \\
\text { teoria del aprendizaje de Ausubel. }\end{array}$ \\
\hline $\begin{array}{l}\text { Nussbaum, 3. y } \\
\text { Novak, J.D., } \\
1976 . \\
\text { Nussbaum, J., } 1979 . \\
\text { Mall, G.B,y } \\
\text { Howe, A., } \\
1979 . \\
\text { Nussbaum, J. y } \\
\text { Sharoni-Dagan, N., } \\
\text { 1983. }\end{array}$ & Noción (notion). & $\begin{array}{l}\text { Constructos conceptuales } \\
\text { realizados por el } \\
\text { investigador inferidos de las } \\
\text { respuestas de los nikios } \\
\text { (Nussbaum y Novak } 1976 \text { ). }\end{array}$ & $\begin{array}{l}\text { Muestra: Nifos de } 2^{\circ} \text { grado. } \\
\text { Contenido: Concepto de Tierra. } \\
\text { Enseñanza audiotutorial (enseñanza } \\
\text { personalizada). Entrevista personal } \\
\text { (2 clases de } 26 \text { alumnos, un grupo } \\
\text { después de la instrucción y el otro, } \\
\text { antes y despues de ia instruccion). }\end{array}$ & $\begin{array}{l}\text { Categoriza respuestas de los alumnos } \\
\text { (dibujos y conversaciones). Las } \\
\text { respuestas, las jerarquiza con el criterio } \\
\text { de acercaniento al concepto cientifico. } \\
\text { Si bien, la taxonomización la obtiene de } \\
\text { las respuestas de los sujetos. Busca } \\
\text { elementos comunes a las respuestas de } \\
\text { los alumnos a las diversis preguntas. } \\
\text { Identiffca versiones cualitativarncnte } \\
\text { diferentes del concepto Tiema sosteridas } \\
\text { por los niños. Las nociones que obtiene } \\
\text { sobre el concepto Tiena (5) son una } \\
\text { aproximación; no tienen por qué ser } \\
\text { representativas de la población y } \\
\text { requieren ser válidas por posteriores } \\
\text { estudios. No realiza estudios } \\
\text { ontogenéticos. }\end{array}$ \\
\hline $\begin{array}{l}\text { Deadman, J. y Kelly, } \\
\text { P.J.. 1978. }\end{array}$ & $\begin{array}{l}\text { Concepciones de los } \\
\text { alumnos } \\
\text { (pupils'conceptions). }\end{array}$ & $\begin{array}{l}\text { Regularidades que se encuentran en } \\
\text { las respuestas de los alumnos a } \\
\text { preguntas realizadas sobre } \\
\text { contenidos cientificos en diferentes } \\
\text { contextos. }\end{array}$ & $\begin{array}{l}\text { Muestra: Alumnos } 11 \text { - } 15 \text { años ( } 52 \\
\text { sujetos), sin ensenanza formal. } \\
\text { Contenido: Herencia y evolución. } \\
\text { Entrevista personal (utiliza } \\
\text { contextos diferentes para estudiar el } \\
\text { mismo concepto). }\end{array}$ & $\begin{array}{l}\text { Categoriza respuestas de los alumnos, a } \\
\text { partir de las cuales tealiza un estudio de } \\
\text { concepciones. Identifica jerarquias } \\
\text { psicológicas de conceptos de forma } \\
\text { similar a Ausubel en lugar de las } \\
\text { jerarquias iogicas de Gagne. Analiza } \\
\text { respuestas de los alumnos desde las } \\
\text { similitudes y discrepancias entre el } \\
\text { conocimiento del alumno y el } \\
\text { academico referido al contenido objeto } \\
\text { de investigacion. Recurre a la historia } \\
\text { de iz ciencia con el fan de encontrar } \\
\text { paralelismos. }\end{array}$ \\
\hline $\begin{array}{l}\text { Novick, S. y } \\
\text { Nussbaum, d., } 1978 .\end{array}$ & $\begin{array}{l}\text { Concepciones de los } \\
\text { alumnos (pupil's } \\
\text { conceptions). }\end{array}$ & $\begin{array}{l}\text { Explicaciones que dan los } \\
\text { alumnos a situaciónes físicas que } \\
\text { pueden ir desde las meras } \\
\text { respuestas de percepción a } \\
\text { modelos más elaborados. } \\
\text { Considera que los sujetos } \\
\text { clasificados en modelos distintos } \\
\text { del científico han asimilado un } \\
\text { modelo distorsionado. }\end{array}$ & $\begin{array}{l}\text { Muestra piloto: } 20 \text { nīos de } 8^{\circ} \\
\text { grado (13-14 años). Muestra dei } \\
\text { estudio: } 3 \text { niños de alto nivel, } 4 \text { de } \\
\text { nivel medio y } 4 \text { de nivel bajo, de } 9 \\
\text { colegios urbanos de } 8^{\circ} \text { grado. } \\
\text { Trabaja cinco aspectos relacionados } \\
\text { con la naturaleza particulada de is } \\
\text { materia. Entrevista fomal. }\end{array}$ & $\begin{array}{l}\text { Categoriza las respuestas de los } \\
\text { alumnos en téminos de los zipos de } \\
\text { explicaciones dadas por dichos } \\
\text { alumnos. }\end{array}$ \\
\hline Albert, E.. 1978. & Concepto (concept). & $\begin{array}{l}\text { Son nociones, } \\
\text { intuiciones y } \\
\text { capacidades cognitivas } \\
\text { que subyacen en sus } \\
\text { conceptualizaciones. }\end{array}$ & $\begin{array}{l}\text { Muestra: Niños de } 4 \text { a } 9 \text { años. } \\
\text { Tema: Calor, metodología } \\
\text { namiralista (similar a la piagetiana). } \\
\text { Entrevistas clinicas de formato fijo } \\
\text { con cuestiones abiertas. }\end{array}$ & $\begin{array}{l}\text { De las respuestas de los sujetos, } \\
\text { identifica modelos de pensamiento, gue } \\
\text { son clasificados tn categorias, de tal } \\
\text { forma que cada categoria contiene } \\
\text { varios modelos, los cuales son } \\
\text { clasificados siguiendo el criterio de } \\
\text { acercamiento progresivo al concepto } \\
\text { científico. Realiza estudio } \\
\text { ontogentético. Planteamiento maturalista } \\
\text { similar al piagetiano. }\end{array}$ \\
\hline Brumby, M.. 1979. & $\begin{array}{l}\text { Conceptos erroneos o } \\
\text { ideas falsas } \\
\text { (misconceptions). }\end{array}$ & $\begin{array}{l}\text { Creencias preexistentes que } \\
\text { bloquean la comprension de los } \\
\text { conceptos bssicos. }\end{array}$ & $\begin{array}{l}\text { Muestra: Estudiantes de ler año de } \\
\text { universidad. Tema: Selección } \\
\text { natural. Tests de respuesta abierta y } \\
\text { de elección múltiple. }\end{array}$ & $\begin{array}{l}\text { Analiza las respuestas desde la ciencia } \\
\text { ( } \mathrm{T}^{\circ} \text { del cambio conceptual y las } \\
\text { categoriza. Afima que las creencias } \\
\text { preexistentes actuan como una bartera } \\
\text { para el aprendizaje formal de un } \\
\text { contenido. }\end{array}$ \\
\hline Brumby, M., 1979. & $\begin{array}{l}\text { Fallos de comprensión } \\
\text { (misunderstandings). }\end{array}$ & $\begin{array}{l}\text { Maia comprensión de los } \\
\text { conceptos. La buena } \\
\text { comprenstón era definida en } \\
\text { términos de los niveles de } \\
\text { aplicación y anślisis de Bloom. }\end{array}$ & $\begin{array}{l}\text { Muestra: Estudiantes de ler año de } \\
\text { universidad. Tema: Selección } \\
\text { natural. Tests de respuesta abierta y } \\
\text { de elección múltiple. }\end{array}$ & $\begin{array}{l}\text { Analiza las respuestas desde la } \\
\text { ciencia (To del cambio conceptual) y } \\
\text { las categoriza. Afima que las } \\
\text { creencias preexistentes actúan cono } \\
\text { una barrera para el aprendizaje } \\
\text { formal de un contenido. }\end{array}$ \\
\hline
\end{tabular}




\begin{tabular}{|c|c|c|c|c|}
\hline AUTORES & SIGNIFICANTE & SIGNIFICADO & CONTEXTO EXIERIMENTAL & CATEGORLZACIÓN \\
\hline $\begin{array}{l}\text { Erickson, G.L., } \\
1979 .\end{array}$ & $\begin{array}{l}\text { Ideas de los niños } \\
\text { (children's ideas). }\end{array}$ & $\begin{array}{l}\text { Son las unidades basicas de análisis } \\
\text { que corresponden a las } \\
\text { explicaciones o justificaciones } \\
\text { realizadas por el nino ante un } \\
\text { problema presentado en el } \\
\text { transcurso de la entrevista. Trenen } \\
\text { carácter personal. Están referidas a } \\
\text { un aspecto concreto de un topico y } \\
\text { se obtienen a partir de las propias } \\
\text { expresiones del sujeto o de lo que } \\
\text { el investigador cree que dice. }\end{array}$ & 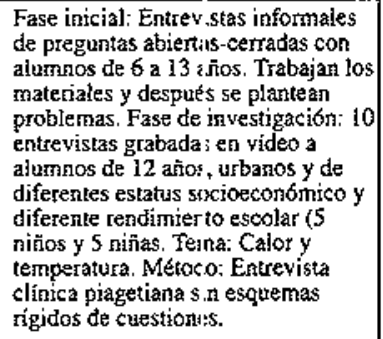 & $\begin{array}{l}\text { Analiza respuestas de los alumnos } \\
\text { desde las similitudes y discrepancias } \\
\text { entre el conocimiento del alumno y el } \\
\text { académico referido al contenido } \\
\text { objeto de investigación. Categoriza } \\
\text { respuestas de los alumnos a partir de } \\
\text { lo cual realiza un estudio de ideas e } \\
\text { ideas compuestas. No realiza } \\
\text { ontogenesis. }\end{array}$ \\
\hline Erickson, G.L., 1979. & $\begin{array}{l}\text { Ideas compuestas de } \\
\text { los nifos (childsen's } \\
\text { composite jdeas). }\end{array}$ & $\begin{array}{l}\text { Lo que de comb́n tienen las ideas } \\
\text { de las niños entrevistados. }\end{array}$ & $\begin{array}{l}\text { Fase inicial: Entrevistas informales } \\
\text { de preguntas abiert is-cerradas con } \\
\text { aibmnos de } 6 \text { a } 13 \text { itnos. Trabajan } \\
\text { los materiales y detpues se plantean } \\
\text { probiemas. Fase de investigacion: } \\
10 \text { entrevistas grab idas en vídeo a } \\
\text { alumnos de } 12 \text { año:, urbanos y de } \\
\text { diferentes estatus s')cioeconómico y } \\
\text { diferente rendimiento escotar ( } 5 \\
\text { niños y } 5 \text { niñas. Te na: Calor y } \\
\text { temperatura. Métorlo: Entrevista } \\
\text { clínica piagetiana sin esqutemas } \\
\text { rigidos de cuestion zs. }\end{array}$ & $\begin{array}{l}\text { Analiza respuestas de los alumnos } \\
\text { desde las similitudes y discrepancias } \\
\text { entre el conocimiento del alumno y el } \\
\text { academico referido al contenido } \\
\text { objeto de investigacion. Categoriza } \\
\text { respuestas de los alumnos a partir de } \\
\text { lo cual realiza un estudio de ideas e } \\
\text { tdeas compuestas. No reajiza } \\
\text { ontogenesis. }\end{array}$ \\
\hline Viemot, L., 1979. & $\begin{array}{l}\text { Razonarniento } \\
\text { espontáneo } \\
\text { (spontaneous } \\
\text { reasoning). }\end{array}$ & $\begin{array}{l}\text { Son esquemas explicativos } \\
\text { comunes a los diferentes alumnos } \\
\text { que realizan la prieba. Se } \\
\text { caracterizan por ser inwitivos. } \\
\text { posiblemente erroneos; persisten a } \\
\text { pesar de la enseñanza y son formas } \\
\text { de pensar utilizadas en la } \\
\text { interacción social que suelen } \\
\text { aparecer incluso en expertos cuando } \\
\text { carecen de tiempo para la reflexión. }\end{array}$ & $\begin{array}{l}\text { Muestra: Alumnos de ultimo curso } \\
\text { de bachillerato y lis curso de } \\
\text { universidad. Contenido: Relaciones } \\
\text { entre fuerza, energia y movimiento. } \\
\text { Pruebas: Test de fapiz y papel. }\end{array}$ & $\begin{array}{l}\text { Analiza respuestas de los alumnos } \\
\text { desde las similitudes y } \\
\text { discrepancias entre el conocimiento } \\
\text { del alumno y el academico referido } \\
\text { al contentdo objeto de } \\
\text { investigacion. Categoriza las } \\
\text { respuestas de los alumnos e } \\
\text { identiftca regularidades. }\end{array}$ \\
\hline Erickson, G.L., 1980. & $\begin{array}{l}\text { Creencias de los } \\
\text { alumnos } \\
\text { (students'beliefs). }\end{array}$ & $\begin{array}{l}\text { Es un juicio de valor de un alumno } \\
\text { acerca de una explicactón dada } \\
\text { sobre una situación física concreta. }\end{array}$ & $\begin{array}{l}\text { Murestra urbana: } 2^{\prime} 6 \text { alumnos } \\
\text { canadienses }\left(5^{\circ}, 7^{\circ} \text { y } 9^{\circ}\right) \\
\text { seleccionados al arar. Tema: Calor y } \\
\text { temperatura. El ini'estigador realiza } \\
\text { una experiencia en clase en la que } \\
\text { los alumnos le observan. Se les } \\
\text { presentan en una pagina, un } \\
\text { conjunto de obser aciones hechas } \\
\text { por los estudiantes durante la } \\
\text { experiencia con di ierentes } \\
\text { explicaciones posisles. El alumno } \\
\text { debe manifestar si preferencia sobre } \\
\text { una escala. }\end{array}$ & $\begin{array}{l}\text { Recurre a la historia de la ciencia. } \\
\text { Análisis cientiffco de los } \\
\text { resultados. Categorizan las } \\
\text { respuestas de los alumnos según } \\
\text { correspondan al modelo cinético, } \\
\text { calorico y punto de vista del } \\
\text { alumno (respurestas expresadas por } \\
\text { estudiantes durante entrevistas } \\
\text { clínicas, Erickson 1979). }\end{array}$ \\
\hline Erickson, G.L., 1980. & $\begin{array}{l}\text { Puntos de vista } \\
\text { (viewpoints). }\end{array}$ & $\begin{array}{l}\text { Es un modelo de perfil conceptual } \\
\text { de los alumnos sobre una } \\
\text { deteminada fenomenología. }\end{array}$ & $\begin{array}{l}\text { Muestra urbanta: } 276 \text { alumnos } \\
\text { canadienses }\left(5^{\circ}, 7^{\circ} \text { y } 9^{\circ}\right) \\
\text { seleccionadios al attar. Tema: Caior } \\
\text { y temperatura. El investigador } \\
\text { realiza una experitncia en clase en } \\
\text { la que los alumnos le observan. Se } \\
\text { les presentan en uila página un } \\
\text { conjunto de abser }: \text { aciones hechas } \\
\text { por los estudiantes durante la } \\
\text { experiencia con diferentes } \\
\text { explicaciones posibles. El alumno } \\
\text { debe manifestar st. preferencia } \\
\text { sobre una escala. }\end{array}$ & $\begin{array}{l}\text { Recurre a la historia de la ciencia. } \\
\text { Analisis científico de los } \\
\text { resultados. Categorizan tas } \\
\text { respuestas de los alumnos según } \\
\text { correspondan al modelo cinético. } \\
\text { calórico y punto de vista del } \\
\text { alumno (respuestas expresadas por } \\
\text { estudiantes durante entrevistas } \\
\text { clínicas, Erickson 1979). }\end{array}$ \\
\hline $\begin{array}{l}\text { Carzmazza, A., } \\
\text { McClosey, M. y } \\
\text { Green, B., } 1981 .\end{array}$ & $\begin{array}{l}\text { Errores conceptales } \\
\text { (misconceptions). }\end{array}$ & $\begin{array}{l}\text { Explicaciones de los alumnos } \\
\text { que son repetibles y } \\
\text { generalizables y que son } \\
\text { utilizados en diferentes } \\
\text { situaciones físicas que difieren } \\
\text { de la explicacion aceptada por } \\
\text { la comunidad cientifica actual. }\end{array}$ & $\begin{array}{l}\text { Muestra: } 50 \text { estud antes } \\
\text { universitarios (10 habian recibido un } \\
\text { curso de física universitaria; } 20 \text {, } \\
\text { enseñanza de fisica en bachillerato; } \\
\text { 14, ningún tipo de enseñanza formal } \\
\text { en física; el resto iro se incluyo en el } \\
\text { análisis por proble mas en el } \\
\text { seguimiento de la: instnicciones del } \\
\text { protocolo). Tema: Cinemática. } \\
\text { Cuestionario de pipel y lápiz. } \\
\text { Análisis de dibujes. }\end{array}$ & $\begin{array}{l}\text { Anałiza las respuestas desde la ciencia } \\
\text { (T' del cambio conceptual y las } \\
\text { categoriza. Afirma que las creencias } \\
\text { preexistentes actúan como una barrera } \\
\text { para el aprendizaje formal de un } \\
\text { contenido. }\end{array}$ \\
\hline $\begin{array}{l}\text { Caramazza, A., } \\
\text { McClosey, M.y } \\
\text { Green, B., } 1981 .\end{array}$ & $\begin{array}{l}\text { Creencias ingenuas } \\
\text { (naive beliefs) o } \\
\text { principios ingenuos } \\
\text { (naive principles). }\end{array}$ & $\begin{array}{l}\text { Son modelos subjetivos de leyes } \\
\text { que reflejan el sistema conceptual } \\
\text { que la gente utiliza para organizar y } \\
\text { realizar inferencias de su } \\
\text { experiencia con el movimiento de } \\
\text { los objetos. }\end{array}$ & $\begin{array}{l}\text { Muestra: } 50 \text { estudiantes } \\
\text { universicarios }(10 \text { habian recibido un } \\
\text { curso de física un: versitaria; } 20 \text {, } \\
\text { enseñanza de física en bachillerato; } \\
14 \text {, ningún tipo de enseinanza fomal } \\
\text { en física; el resto ro se incluyo en el } \\
\text { anátisis por problumas en el } \\
\text { seguimiento de las instrucciones del } \\
\text { provocolo). Tema: Cinemática. } \\
\text { Cuestionario de papel y lápiz. } \\
\text { Análisis de dibujos. }\end{array}$ & $\begin{array}{l}\text { Analiza las respuestas desde la ciencia } \\
\text { (T" del cambio conceptual) y las } \\
\text { categoriza. Afirma que las creencias } \\
\text { preexistentes actuan como una barrera } \\
\text { para el aprendizaje formal de un } \\
\text { contenido. }\end{array}$ \\
\hline
\end{tabular}




\begin{tabular}{|c|c|c|c|c|}
\hline AUTORES & SIGNIFICANTE & SIGNIFICADO & CONTEXTO EXPERIMENTAL & CATEGORIZACION \\
\hline $\begin{array}{l}\text { Novick, S. y } \\
\text { Nussbaum, J., } 1981 \text {. }\end{array}$ & Preconcepción. & $\begin{array}{l}\text { Explicaciones verbales o icónicas } \\
\text { que da un sujeto a una situación } \\
\text { fisica deteminada. }\end{array}$ & $\begin{array}{l}\text { Muestra: Desde alumnos } \\
\text { estadounidenses que provienen de la } \\
\text { escuela elemental hasta } \\
\text { universitarios de } 2^{\circ} \text { año no } \\
\text { especializados en ciencias ( } 576 \\
\text { sujetos). Tema: Naturaleza } \\
\text { particulada de la materia. Tareas de } \\
\text { papel y lapiz (test sobre partículas } \\
\text { sacado en un estudio anterior de } \\
\text { entrevistas, Novick y Nussbaum } \\
\text { 1978). }\end{array}$ & $\begin{array}{l}\text { Categoriza las respuestas de los } \\
\text { alumnos (preconcepciones). Corupone } \\
\text { respuestas de los alumnos a eravés de } \\
\text { la edad para poner de manifiesto } \\
\text { dificultades cognitivas. Analiza desde } \\
\text { la ciencia y añade aspectos } \\
\text { psicológicos como la intuicion o la } \\
\text { imagnación para explicar que existen } \\
\text { explicaciones de los sujetos } \\
\text { alternativas a la de la ciencia. }\end{array}$ \\
\hline Driver, R., 1981. & $\begin{array}{l}\text { Esquemas alternativos } \\
\text { de los alumnos } \\
\text { (pupils' alternative } \\
\text { frameworks). }\end{array}$ & $\begin{array}{l}\text { Son creencias que tienen los } \\
\text { alumnos sobre el modo en que } \\
\text { ocurren los fenómenos naturales } \\
\text { y que afectan a la forma de } \\
\text { entender las experiencias que se } \\
\text { presentan en el aula. Son } \\
\text { consecuencia de la creatividad e } \\
\text { imaginación del sujeto junto con } \\
\text { una experiencia directa y de la } \\
\text { interacción social. }\end{array}$ & $\begin{array}{l}\text { Muestra: Sujetos entre } 11 \text { y } 13 \\
\text { años. Tema: Fuerza, calor y } \\
\text { dilatacion. Se graban respuestas de } \\
\text { dos o tres sujetos acerca de } \\
\text { determinadas experiencias, durante } \\
\text { la actuacion didactica. }\end{array}$ & $\begin{array}{l}\text { Analiza desde la ciencia y añade } \\
\text { aspectos psicologicos como la } \\
\text { intuición o la imaginación para } \\
\text { explicar que existen explicaciones de } \\
\text { los sujetos alternativas a la de la } \\
\text { ciencia. No categoriza resplestas. }\end{array}$ \\
\hline $\begin{array}{l}\text { Nussbaum, J. y } \\
\text { Sharoni-Dagan, N., } \\
1983 .\end{array}$ & $\begin{array}{l}\text { Conceptos erróneos o } \\
\text { ideas falsas } \\
\text { (misconceptions). }\end{array}$ & $\begin{array}{l}\text { Son preconcepciones } \\
\text { sustancialmente diferentes a la } \\
\text { noción cientifica aceptada y que } \\
\text { inhiben el aprendizaje. }\end{array}$ & $\begin{array}{l}\text { Nifios de } 2^{\circ} \text { grado de un colegio } \\
\text { público elemental de Jemusalen de } \\
\text { estatus socioeconónico bajo y } \\
\text { medio-alio. Tema: La Tierra como } \\
\text { cuerpo cósnico. Unidad } \\
\text { audiocultural. Tres muestras: A ( } 41) \text {, } \\
\text { pretest, leccion audiotutorial y } \\
\text { postest; B (37), lección audiotutorial } \\
\text { y postest; C (36), postest. }\end{array}$ & $\begin{array}{l}\text { Utiliza el término preconcepción } \\
\text { haciéndolo sinónimo a estucturas } \\
\text { alternativas y el de misconceptions } \\
\text { a nociones relevantes de ies niños. } \\
\text { Considera que las nociones son } \\
\text { juicios cognitivos con los que se } \\
\text { caracteriza categorialmente ia } \\
\text { estructura cognitiva de los } \\
\text { estadiantes. En sus planteamientos } \\
\text { téricos está influenciado por } \\
\text { Ausubel; tarnbítn acepta la vision } \\
\text { de Driver y Fasely (1978) que } \\
\text { consideraba que los aprendices } \\
\text { tenden a construir concepciones o } \\
\text { estructuras ailernativas. }\end{array}$ \\
\hline $\begin{array}{l}\text { Carrascosa Alis, J. y } \\
\text { Gisil Pérez, D., } 1985 .\end{array}$ & Preconcepto. & $\begin{array}{l}\text { Son conceptos distintos de los } \\
\text { científicos. }\end{array}$ & $\begin{array}{l}\text { Muestra: } 145 \text { alumnos de COU, } 136 \\
\text { alumios de CAP y } 145 \text { profesores } \\
\text { de secundaria en aclivo. } \\
\text { Cuestionatio de papel y lapiz sacado } \\
\text { de las respuestas erroneas obtentdas } \\
\text { en clase de alumnos de BUP. }\end{array}$ & $\begin{array}{l}\text { Analiza las respuestas de los } \\
\text { alumnos desde las similitudes y } \\
\text { discrepancias entre el conocimiento } \\
\text { del alumno y el académico referido } \\
\text { al contenido objeto de investigación. } \\
\text { Son consecuencia de la aplicación } \\
\text { de la melodología de la } \\
\text { superficialidad. Para su } \\
\text { modificación se exige un cambio } \\
\text { metodologico. }\end{array}$ \\
\hline $\begin{array}{l}\text { Meheut, M. Saltiel, } \\
\text { E. y Tiberghien, A., } \\
1985 \text {. }\end{array}$ & $\begin{array}{l}\text { Conceptos erróneos o } \\
\text { ideas falsas } \\
\text { (misconceptions). }\end{array}$ & $\begin{array}{l}\text { Son constractos realizados por el } \\
\text { investigador para referirse a los } \\
\text { esquemas explicativos de los } \\
\text { sujetos que difieren de los } \\
\text { aceptados por la comunidad } \\
\text { cientifica. }\end{array}$ & 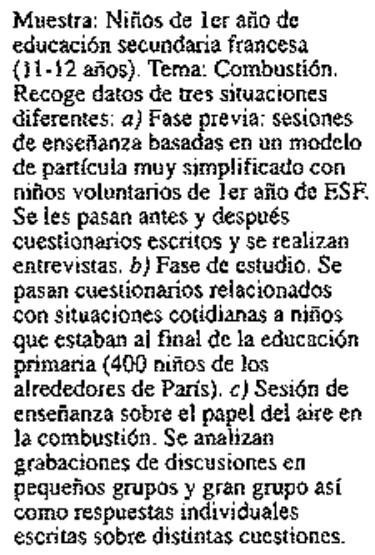 & $\begin{array}{l}\text { Analiza respuestas de los alumnos } \\
\text { desde las similitudes y } \\
\text { discrepancias entre el conocimiento } \\
\text { del alumno y el academico referido } \\
\text { al contenido objeto de } \\
\text { investigacion. }\end{array}$ \\
\hline
\end{tabular}




\begin{tabular}{|c|c|c|c|c|}
\hline AUTORES & SIGNIFICANTE & SIGNIFICADO & CONTEXTO EIPERJMENTAL & CATEGORIZACIÓN \\
\hline $\begin{array}{l}\text { Meheut, M., Salciel, } \\
\text { E. y Tiberghien, A.4 } \\
1985 .\end{array}$ & $\begin{array}{l}\text { Esquemas explicativos } \\
\text { (explanatory } \\
\text { frameworks). }\end{array}$ & $\begin{array}{l}\text { Explicaciones de los alumnos a una } \\
\text { serie de situaciones fisicas } \\
\text { cotidianas que tienen ciento carácter } \\
\text { de estabilidad. }\end{array}$ & 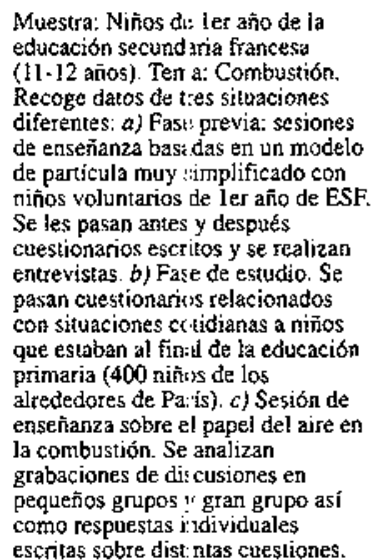 & $\begin{array}{l}\text { Analiza las respuestas desde la ciencia } \\
\text { ( } T^{\circ} \text { del cambio conceprual) y las } \\
\text { categoriza. Afirma que las creencias } \\
\text { preexistentes actúan como una barrera } \\
\text { para el aprendizaje formal de un } \\
\text { contenido. }\end{array}$ \\
\hline
\end{tabular}

